\title{
Simulation of the Flow Field Characteristics of a Downburst
}

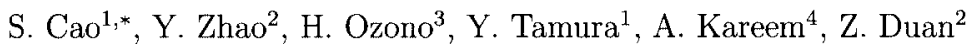

1 Wind Engineering Research Center, Tokyo Polytechnic University, Tokyo 243-0297, Japan

${ }^{2}$ Department of Civil Engineering, Harbin Institute of Technology, Harbin 150090, China

${ }^{3}$ Department of Physics, Miyazaki University, Miyazaki 889-2155, Japan

${ }^{4}$ Department of Civil Engineering and Geological Sciences, University of Notre Dame, IN 46556, USA

Email: cao@arch.t-kougei.ac.jp

\begin{abstract}
Wind load on a structure is usually investigated in turbulent boundary layer flow simulated in a boundary layer wind tunnel. However, the strong wind that causes damages on structures is usually transient wind, for instance, downburst wind, whose statistics are different with the general atmospheric boundary layer. Thus, the investigation of wind- reduced force on structures in transient flow is very important at the point of wind hazard mitigation. In this study, we tried to model the turbulence statistics of a downburst boundary layer in an actively-controlled wind tunnel which has 99 fans controlled individually through a computer[1]. The flow, at the beginning, is controlled to be general boundary layer type. A sudden abrupt velocity change was then introduced into the flow which has a positive shear on the lower part and minus shear in the upper part of the boundary layer[2]. Although the velocity profile is actually time-dependent after acceleration and will recover to general boundary layer type finally, we considered constant velocity profile after acceleration. The reason is that at first there is no successful measurement of the downburst which can be used as target for simulation. Another reason is that the stronger shear is possibly more unfavorable to the structures. In this paper, we will describe the flow-generating technique and study on the characteristics of the simulated flow.
\end{abstract}

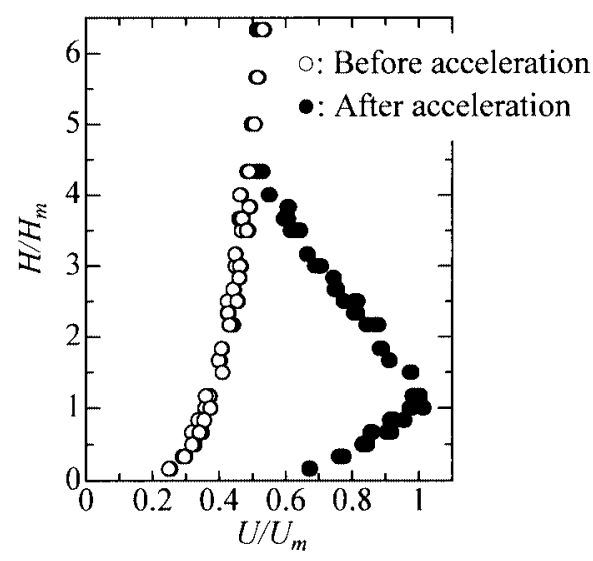

Figure: Vertical profile of mean velocity

\section{REFERENCES}

1. Oseguera RM, Bowles RL. A simple analytic 3-dimentioanl downburst model based on boundary layer stagnation flow. NASA Technical Memorandum 100632, July 1988

2. Cao S, Nishi A, Kikugawa H, Matsuda Y. Reproduction of wind velocity in a multiple fan wind tunnel. Journal of Wind Engineering and Industrial Aerodynamics, 2002; 90: 1719-1729 